\title{
Modelling the spectral evolution of classical double radio sources
}

\author{
K. Manolakou^ and J. G. Kirk \\ Max-Planck-Institut für Kernphysik, Saupfercheckweg 1, 69117 Heidelberg, Germany \\ e-mail: Dina.Manolakou@mpi-hd.mpg.de
}

Received 1 March 2002 / Accepted 17 May 2002

\begin{abstract}
The spectral evolution of powerful double radio galaxies (FR II's) is thought to be determined by the acceleration of electrons at the termination shock of the jet, their transport through the bright head region into the lobes and the production of the radio emission by synchrotron radiation in the lobes. Models presented to date incorporate some of these processes in prescribing the electron distribution which enters the lobes. We have extended these models to include a description of electron acceleration at the relativistic termination shock and a selection of transport models for the head region. These are coupled to the evolution of the electron spectrum in the lobes under the influence of losses due to adiabatic expansion, by inverse Compton scattering on the cosmic background radiation and by synchrotron radiation. The evolutionary tracks predicted by this model are compared to observation using the power/source-size $(P-D)$ diagram. We find that the simplest scenario, in which accelerated particles suffer adiabatic losses in the head region which become more severe as the source expands produces $P-D$-tracks which conflict with observation, because the power is predicted to decline too steeply with increasing size. Agreement with observation can be found by assuming that adiabatic losses are compensated during transport between the termination shock and the lobe by a re-acceleration process distributed throughout the head region.
\end{abstract}

Key words. acceleration of particles - plasmas - shock waves - galaxies: active - galaxies: high redshift - galaxies: jets

\section{Introduction}

Powerful double radio galaxies or "classical doubles" (CDs) owe their name to the extended (hundreds of kpc) lobes of radio emission they exhibit on opposite sides of the parent galaxy. Fanaroff \& Riley (1974) classified these sources as type II objects; they have luminosities $P_{178 \mathrm{MHz}}>5 \times 10^{25} \mathrm{~W} \mathrm{~Hz}^{-1}$ and are edge-brightened, with bright outer hotspots. It is universally agreed that the radio continuum of the CDs is synchrotron radiation from relativistic electrons and perhaps positrons. The standard scenario is that of a jet propagating from the galaxy to the outer parts of the lobes, passing through a shock front at the hotspots and subsequently filling a "cocoon" around the jet with radiating particles - see, for example, Begelman \& Cioffi (1989); Peacock (1999).

Falle (1991) considered radio sources as expanding bubbles that are fed by supersonic jets and drive a bow shock into a radially stratified external medium. He showed that the jet length and the bow shock grow in a self-similar way for external atmospheres in which the density drops off more slowly than $1 / r^{2}$ from the centre of the galaxy. Subsequently,

Send offprint requests to: J. G. Kirk, e-mail: John.Kirk@mpi-hd.mpg.de

* On leave from the Department of Physics, University of Thessalonica.
Kaiser \& Alexander (1997) showed that the cocoons also expand self-similarly. Based on this result, Kaiser et al. (1997) developed an analytical model for the spectral evolution of FR II sources as a function of redshift, jet power and the scaling of the external density profile. They assumed that electrons with an initial power-law distribution in energy are continuously injected into the plasma immediately downstream of the shock front that terminates the jet. A key point of their treatment is that the pressure in this region equals that which drives the bow shock into the external medium on the axis of the source. This results from the fact that the jet is confined by the pressure of the cocoon (Begelman \& Cioffi 1989), so that the working surface over which its thrust is distributed expands self-similarly, along with the bow shock and cocoon. Particles undergo adiabatic losses on moving from the terminal shock front into the main part of the cocoon, whose pressure is lower than that at the working surface by a constant factor (taken as $\approx 16$ by Kaiser et al. 1997). They then undergo synchrotron and inverse Compton losses, as well as adiabatic losses as the lobe of the source expands. Using this model, Kaiser et al. (1997) computed evolutionary tracks in the power-linear size $(P-D)$ plane. These display decreasing power as the size increases, in agreement with observations, which indicate a deficit of large luminous sources. 


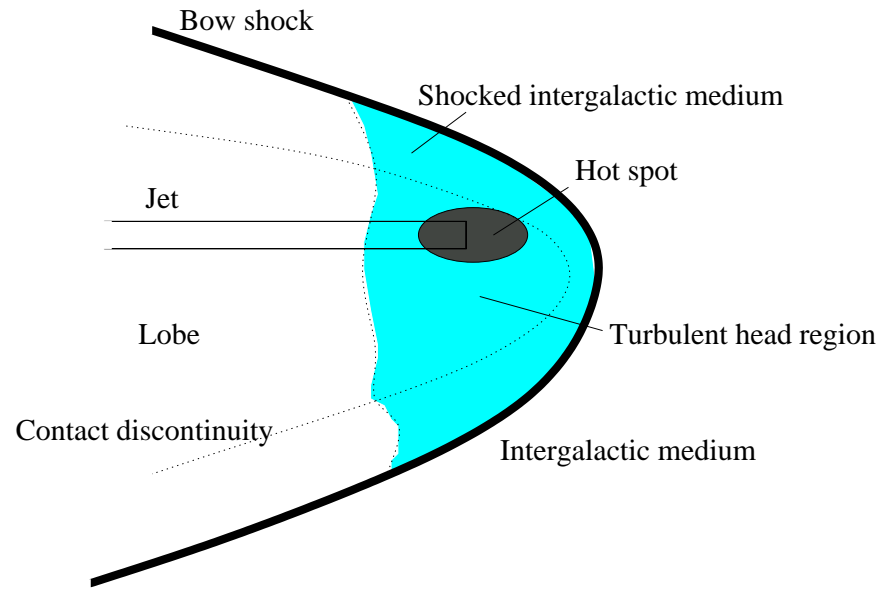

Fig. 1. A cartoon of the interaction of the intergalactic medium and a jet of an FR II radio galaxy, showing the principal components of the spectral evolution model. A jet of constant Lorentz factor and power is decelerated in a hot spot (whose size is independent of source age) converting part of its energy into relativistic electrons and part into magnetic field. The electrons propagate according to a prescribed transport model (parameterised by $\alpha$ and $\tau$ - see Eq. (18)) from the hotspot into the turbulent head region. The hot spot moves around inside the head region; averaged over time, the jet thrust is balanced by the pressure in the head region, which expands self-similarly along with the bow shock and lobe. Synchrotron losses are important in the (constant) magnetic field of the hot spot, but adiabatic losses suffered in moving into the head region and later into the lobe may be compensated by a re-acceleration process acting in either the hot spot or the head region. Once in the lobe, time-dependent synchrotron, inverse Compton and adiabatic losses determine the spatially integrated electron spectrum.

However, Blundell et al. (1999) noted that there is no evidence that the size of the working surface is proportional to the size of the source. Instead, the hot-spots of all known FR II sources appear to be a few kiloparsecs in diameter. This has a profound influence on the predicted evolutionary tracks, because it implies that the adiabatic losses suffered upon moving into the cocoon by particles accelerated at the termination shock are not constant, but increase strongly with the age of the source. Blundell et al. (1999) modelled the spectral evolution of CDs including this effect by prescribing the spectrum of electrons entering the cocoon to be a broken power-law distribution. They, too, found evolutionary tracks that agree with the observed lack of large, luminous radio sources, as well as with several other properties of the samples they investigated.

In this paper we present computations of evolutionary tracks based on the picture adopted by Blundell et al. (1999). However, instead of prescribing the electron distribution that enters the cocoon, we assume that electrons are accelerated by the first-order Fermi process at the termination shock and then propagate through the hot spot region into the cocoon or "lobe" according to one of two models, which we designate case A and case B. In case A, the full adiabatic energy loss corresponding to the age dependent pressure difference between the hot spot and cocoon is applied. This results in $P-D$ tracks which are in conflict with the observations. One possible way out of this problem is to assume that a re-acceleration process occurs after the initial encounter with the termination shock. Such a process is indicated independently by investigations of the spectra of individual hot-spots and of optical synchrotron emitting jets (Meisenheimer et al. 1996, 1997; Perley et al. 1997; Wagner \& Krawczynski 2000). This motivates our case B model, in which we assume that the adiabatic losses between the termination shock and the lobe are compensated by a reacceleration process during propagation through the head region. In both cases electrons are carried through the high-loss region by fluid elements whose residence time is distributed according to a specified transport equation. During this time they suffer synchrotron and inverse Compton losses. After entering the cocoon, we follow the particle distribution as it cools and radiates and compute evolutionary tracks as well as snapshots of the spectrum at different ages.

The paper is organised as follows: in Sect. 2 we summarise the hydrodynamic picture which we adopt for the source evolution. In Sect. 3 our treatment of Fermi acceleration is described, the transport model is specified and the kinetic equation obeyed by particles in the cocoon is formulated. Section 4 is devoted to a series of tests and special cases to illustrate the way in which the transport and acceleration models influence the particle distribution in the hot spot and the lobe. Our main results on spectral evolution are presented in Sect. 5, where we conclude that the observed data exclude case A i.e., they can be explained only by a model such as case B, that includes re-acceleration of electrons after their encounter with the termination shock. This conclusion and our results on source spectra at different ages are discussed and compared with previous work in Sect. 6.

\section{Hydrodynamic model}

The standard model for the CD radio sources comprises a central object (AGN) from which emanate two jets in opposite directions, embedded in a cocoon or lobe of diffuse emission. The jets themselves terminate in regions of intense emission, called the hotspots or working surfaces, after which the shocked jet material flows into the cocoon. This material drives a bow shock into the external medium, from which it is separated by a contact discontinuity. A cartoon of the model is shown in Fig. 1.

Following Blundell et al. (1999), we assume that the radial profile of the external (intergalactic) medium does not vary with redshift and scales with distance $r$ from the centre of the host galaxy as

$\rho=\rho_{\mathrm{c}}\left(r / r_{\mathrm{c}}\right)^{-\beta}$

(Garrington \& Conway 1991; Mulchaey \& Zabludoff 1998). Typical values for the above parameters are: $\rho_{\mathrm{c}}=1.67 \times$ $10^{-23} \mathrm{~kg} \mathrm{~m}^{-3}, r_{\mathrm{c}}=10 \mathrm{kpc}$ and $\beta=1.5$.

During the lifetime of the source, we assume that the power $Q_{0}$ carried by the jet and its Lorentz factor $\Gamma_{\text {jet }}$ remain constant. The jet terminates at a shock front which we identify as the primary hotspot embedded in a high-pressure region at the end of the lobe which, following the terminology of Blundell et al. (1999), we call the head region. The exact position of the termination shock within this region is assumed to vary in the manner proposed by Scheuer (1982) in the "dentist's drill 
model". Averaged over time, the thrust of the jet is distributed over the head region, which itself expands with the rest of the source. However, the size of the primary hotspot is independent of source age. Although its ram pressure substantially exceeds the average pressure in the head, simulations indicate that it does not advance much more rapidly (Cox et al. 1991), because it quickly encounters material of higher than average density. Consequently, the pressure $p_{\mathrm{h}}$ immediately downstream of the termination shock can be estimated as the jet thrust, $Q_{0} / c$, divided by the area, $A$, of the primary hotspot itself. Assuming the jet is relativistic and the magnetic field is amplified to reach equipartition with the thermal energy density, we may express the (constant) magnetic energy density in the hotspot, $u_{\mathrm{h}}$, in terms of the jet power as follows:

$u_{\mathrm{h}} \sim \frac{3}{2} p_{\mathrm{h}}=\frac{3}{2} \frac{Q_{0}}{c A}$.

Falle (1991) and Komissarov \& Falle (1998), found solutions in which the size of a radio source increases in a self-similar way with time, with the total length scaling as:

$D(t)=2 c_{1}\left(\frac{t^{3} Q_{0}}{\rho_{\mathrm{c}} r_{\mathrm{c}}^{\beta}}\right)^{1 /(5-\beta)}$

From the measured expansion speeds of powerful sources one concludes that $c_{1} \approx 1.8$ (Blundell et al. 1999).

In contrast to the constant volume and magnetic field of the hotspots, the lobes of the source expand self-similarly. Applying the jump conditions at the external bow shock and using Eq. (3), gives for the pressure of the downstream plasma immediately behind the bow shock:

$p_{\mathrm{d}}(t)=\frac{18}{\Gamma_{\mathrm{x}}+1} \frac{c_{1}^{(2-\beta)}}{(5-\beta)^{2}}\left(\rho_{\mathrm{c}} r_{\mathrm{c}}^{\beta}\right)^{3 /(5-\beta)} Q_{0}^{(2-\beta) /(5-\beta)} t^{-(4+\beta) /(5-\beta)}$

(Kaiser \& Alexander 1997) where $\Gamma_{\mathrm{x}}$ is the ratio of specific heats of the external gas, which we take to be $5 / 3$.

The pressure in the lobes of the source, $p_{\text {lobe }}$, is somewhat lower than this value, depending on the axial ratio of the source. In this way the pressure gradient is provided to drive a high speed back-flow from the head towards the core of the galaxy (Liu et al. 1992). Assuming, in addition, that the magnetic field is tangled and that equipartition is also attained in the lobes, leads to the conclusion that the magnetic energy density in the lobe, $u_{\text {lobe }}$, scales as:

$u_{\text {lobe }}(t)=u_{\mathrm{h}}\left(\frac{t}{t_{0}}\right)^{-a}$

where $a=(4+\beta) /(5-\beta)$ and $t_{0}$ is the time at which the size of the head region was comparable to that of the primary hot spot:

$t_{0}=\left[\frac{3}{\Gamma_{\mathrm{x}}+1} \frac{c_{1}^{2-\beta}}{(5-\beta)^{2}} c A\right]^{1 / a}\left(\frac{\rho_{\mathrm{c}} r_{\mathrm{c}}{ }^{\beta}}{Q_{0}}\right)^{3 /(4+\beta)}$.

\section{Particle acceleration and transport}

We assume that the first-order Fermi process operates at the termination shock in the jet and accelerates there electrons (and possibly positrons) into a distribution which can be described by a power-law in energy. As they move away from the shock these electrons radiate and form the primary hot spot within the head region. We can consider this process as an injection of electrons at a rate $Q_{\mathrm{s}}(\gamma)$ into the plasma behind the termination shock:

$Q_{\mathrm{s}}(\gamma)= \begin{cases}q_{0} \gamma^{-p} & \text { for } \gamma_{\min } \leq \gamma \leq \gamma_{\max } \\ 0 & \text { otherwise. }\end{cases}$

If the jet Lorentz factor $\Gamma_{\text {jet }}$ is of the order of 10, we can choose $2<p<2.3$ (Kirk et al. 2000; Achterberg et al. 2001). The upper and lower limits on the Lorentz factor $\gamma_{\max }$ and $\gamma_{\min }$ are not available from theory. It is plausible to assume $\gamma_{\min } \approx \Gamma_{\text {jet }}$, but $\gamma_{\max }$ should be determined by a local balance of the acceleration and cooling rates. Fortunately, our models are not sensitive to this parameter, since synchrotron cooling within the head region will in any case determine the effective maximum energy of those particles which enter the lobe. The fraction of the jet power, $\eta Q_{0}$, (with $\eta<1$ ) which is transfered into accelerated particles at the termination shock is $\int \gamma m_{\mathrm{e}} c^{2} Q_{\mathrm{s}} \mathrm{d} \gamma$. By means of Eq. (7) this gives for $q_{0}$ :

$q_{0}=\frac{\eta Q_{0}}{m_{\mathrm{e}} c^{2}}(p-2)\left(\gamma_{\min }^{2-p}-\gamma_{\max }^{2-p}\right)^{-1}$

After injection into the primary hot spot, electrons are transported by the turbulent motions of the plasma through the head, and eventually reach the lobes. During this time, which we assume is short compared to the lifetime of the source, they are subject to synchrotron losses in the relatively strong magnetic field generated behind the shock as well as inverse Compton losses by scattering off the cosmic background radiation. The effect which this has on the power-law distribution injected at the termination shock depends on the distribution of the "escape time" i.e., on the probability an injected particle has of leaving the region of strong field after a certain time.

This distribution can be modelled in several ways. The simplest approach would be to assume each particle escapes after a fixed time, corresponding, for example, to the time taken for a fluid element to cross the head region at a given speed. Upon reaching the lobes, the particles would then have the distribution computed by Kardashev (1962), which vanishes above a cut-off energy at which the escape time equals the cooling time. However, the turbulent appearance of the hot spot and head regions indicates that not all elements of shocked plasma will take the same time to escape into the lobes. At the next level of sophistication, this can be modelled in a diffusion picture - a plasma blob, and along with it accelerated particles, performs a random walk through the head region. A convenient formalism, which has been widely used to describe transport in turbulent fluids and other random media (Bouchaud \& Georges 1990 ) is given by the theory of continuous time random walks (see Ragot \& Kirk 1997 and references therein). Diffusion in space results if a sequence of jumps of fixed length $\sigma$ in a random direction is performed, where each jump takes the same time $\tau_{\mathrm{D}}$. The advantage of this formalism is that by changing the waiting time distribution between jumps it is easily generalised to include non-diffusive or "anomalous" transport characterised by a single transport parameter $\alpha$. The mean square 
distance $\left\langle\Delta r^{2}\right\rangle$ of a particle from its starting point is linearly proportional to the elapsed time only in the special case of diffusive transport $\alpha=1$; in general one has $\left\langle\Delta r^{2}\right\rangle \propto t^{\alpha}$ with $0<\alpha<2$. Sub-diffusive transport $(\alpha<1)$ occurs when the motion of the fluid elements is confined in some way - for example to forwards and backwards motion in a channel, which itself diffuses sideways. Supra-diffusive transport, on the other hand, corresponds to a more "free-streaming"-like motion, less impeded by the random eddies. The spatial propagator for the generalised transport process in spherical geometry is

$$
\begin{aligned}
P(r, t)= & {\left[\frac{3}{2}\left(\frac{\alpha \tau_{\mathrm{D}}}{t}\right)^{\alpha}\left(\frac{\sigma}{r}\right)^{2(1-\alpha)}\right]^{3 /[2(2-\alpha)]} \frac{\sqrt{2-\alpha}}{\sigma^{3} \pi^{3 / 2}} } \\
& \times \exp \left\{-(2-\alpha)\left[\frac{3 r^{2}}{2 \sigma^{2}}\left(\frac{\alpha \tau_{\mathrm{D}}}{t}\right)^{\alpha}\right]^{1 /(2-\alpha)}\right\}
\end{aligned}
$$

where $\tau_{\mathrm{D}}$ is a characteristic timescale for the jumps.

During propagation, the rate at which an electron of energy $\gamma(t) m_{\mathrm{e}} c^{2}$ loses energy is described by the equation:

$$
\frac{\mathrm{d} \gamma}{\mathrm{d} t}=-g \gamma^{2}, \quad g=\frac{4}{3} \frac{\sigma_{\mathrm{T}}}{m_{\mathrm{e}} c}\left(u_{\mathrm{h}}+u_{\mathrm{c}}\right)
$$

where $\sigma_{\mathrm{T}}, m_{\mathrm{e}}$ and $c$ are the Thomson cross section, the electron rest mass and the speed of light, respectively. The energy density $u_{\mathrm{c}}$ of the cosmic background radiation field is given in terms of an effective magnetic field $B_{\mathrm{c}}$ by

$u_{\mathrm{c}}=\frac{B_{\mathrm{c}}^{2}}{2 \mu_{0}}, \quad B_{\mathrm{c}}=0.318(1+z)^{2} \mathrm{nT}$

and is taken to be constant during the lifetime of a radio source. Equation (10) may be integrated to give $\gamma^{-1}(t)=\gamma^{-1}\left(t^{\prime}\right)+(t-$ $\left.t^{\prime}\right) g$. These loss processes may be included on the right-hand side of Eq. (9), leading to a composite propagator $\bar{P}\left(\mathbf{x}, t, \gamma, \gamma^{\prime}\right)$ that expresses the probability of finding a particle with Lorentz factor $\gamma$ at $\mathbf{x}$ and $t$, given that it started at the origin $(\mathbf{x}=0$, $t=0)$ with Lorentz factor $\gamma^{\prime}$ :

$$
\bar{P}\left(\mathbf{x}, t, \gamma, \gamma^{\prime}\right)=P(\mathbf{x}, t) \delta\left[\gamma-\frac{\gamma^{\prime}}{1+t g \gamma^{\prime}}\right] .
$$

The distribution $N_{R}(\gamma)$ in Lorentz factor of the total number of particles contained in a sphere of radius $R$, at the centre of which particles are injected according to Eq. (7) starting at time $t_{\text {start }}$ is

$$
N_{R}(\gamma)=4 \pi \int_{0}^{R} \mathrm{~d} r r^{2} \int_{\gamma}^{\infty} \mathrm{d} \gamma^{\prime} \int_{t_{\mathrm{start}}}^{t} \mathrm{~d} t^{\prime} \bar{P}\left(r, t-t^{\prime}, \gamma, \gamma^{\prime}\right) Q_{\mathrm{s}}\left(\gamma^{\prime}\right)
$$

The two timescales that determine $N_{R}$ are the transport timescale (a generalisation of the diffusion time)

$$
t_{\text {diff }}=(2-\alpha)^{(1-\alpha) / \alpha}\left(\frac{3(2-\alpha) R^{2}}{2 \sigma^{2}}\right)^{1 / \alpha} \alpha \tau_{\mathrm{D}}
$$

and the cooling time, defined for a particle of Lorentz factor $\gamma_{\min }$ as $t_{\text {cool }}=1 /\left(g \gamma_{\min }\right)$. If these are both short compared to the age of the source, it is reasonable to assume the transport process into the lobes reaches a steady state, found by setting $t_{\text {start }} \rightarrow-\infty$ :

$$
N_{R}(\gamma)=\left\{\begin{array}{cc}
0 \quad & \text { for } \gamma>\gamma_{\max } \\
\frac{4 \pi q_{0}}{g \gamma^{2}} \int_{\gamma}^{\gamma_{\max }} \mathrm{d} \gamma^{\prime} \gamma^{\prime-p} \int_{0}^{R} \mathrm{~d} r r^{2} P(r, s) \\
\quad \text { for } \gamma_{\min }<\gamma<\gamma_{\max } \\
\frac{4 \pi q_{0}}{g \gamma^{2}} \int_{\gamma_{\min }}^{\gamma_{\max }} \mathrm{d} \gamma^{\prime} \gamma^{\prime-p} \int_{0}^{R} \mathrm{~d} r r^{2} P(r, s) \\
\text { for } \gamma<\gamma_{\min }
\end{array}\right.
$$

where $s=\left(\gamma^{\prime}-\gamma\right) /\left(g \gamma \gamma^{\prime}\right)$. The kinetic equation satisfied by $N_{R}$ reads

$\frac{\partial N_{R}}{\partial t}-\frac{\partial}{\partial \gamma}\left(g \gamma^{2} N_{R}\right)=Q_{\mathrm{s}}(\gamma)-Q_{\mathrm{h}}(\gamma)$

where $Q_{\mathrm{h}}(\gamma)$ is the rate at which particles leave the sphere of radius $R$ (and so enter the lobe). In the steady state, it is straightforward to solve Eq. (16) for $Q_{\mathrm{h}}$. The result is conveniently expressed as a modulation function $M$ describing the effects of cooling on the incident distribution $Q_{\mathrm{s}}$, and defined as

$Q_{\mathrm{h}}(\gamma)=q_{0} \gamma^{-p} M\left(\hat{\gamma}, \rho_{\mathrm{d}}, p, \tau\right)$

where we have also defined parameters representing the Lorentz factor: $\hat{\gamma}=\gamma / \gamma_{\min }$, the reciprocal of the dynamic range of the incident distribution: $\rho_{\mathrm{d}}=\gamma_{\min } / \gamma_{\max }$ and the dimensionless ratio of the transport time to the cooling time of a particle at $\gamma_{\text {min }}: \tau=t_{\text {diff }} / t_{\text {cool }}$. From Eq. (16) we find

$$
M\left(\hat{\gamma}, \rho_{\mathrm{d}}, p, \tau\right)=\left\{\begin{array}{l}
0 \quad \text { for } \hat{\gamma}>1 / \rho_{\mathrm{d}} \\
\frac{2}{\sqrt{\pi}} \int_{x_{1}}^{\infty} \mathrm{d} x x^{1 / 2}\left[1-\frac{\tau \hat{\gamma}}{x^{(2-\alpha) / \alpha}}\right]^{p-2} \mathrm{e}^{-x} \\
\quad \text { or } 1<\hat{\gamma}<1 / \rho_{\mathrm{d}} \\
\frac{2}{\sqrt{\pi}} \int_{x_{1}}^{x_{2}} \mathrm{~d} x x^{1 / 2}\left[1-\frac{\tau \hat{\gamma}}{x^{(2-\alpha) / \alpha}}\right]^{p-2} \mathrm{e}^{-x} \\
\quad \text { for } \hat{\gamma}<1
\end{array}\right.
$$

where

$$
\begin{aligned}
& x_{1}=\left[\frac{\hat{\gamma} \tau}{\left(1-\rho_{\mathrm{d}} \hat{\gamma}\right)}\right]^{\alpha /(2-\alpha)} \\
& x_{2}=\left[\frac{\hat{\gamma} \tau}{(1-\hat{\gamma})}\right]^{\alpha /(2-\alpha)} .
\end{aligned}
$$

Note that transport from the primary hot spot into the lobes results from the diffusion of plasma elements in a turbulent environment. The mean free paths involved are much larger than those estimated for individual energetic electrons, which are usually taken to be of the order of the gyro radius. The turbulent mean free path is also independent of the particle energy. However, this expression still does not account either for adiabatic losses or for any acceleration process that might occur between leaving the termination shock and entering the lobe.

The effect of adiabatic losses is to reduce the energy of each particle by a factor given by the ratio of the pressures before and after the expansion (see, for example, Scheuer \& Williams 1968). In the case of a transition from the pressure in the primary hot spot to the pressure in the lobe we have 
$\gamma \rightarrow\left(u_{\text {lobe }} / u_{\mathrm{h}}\right)^{1 / 4} \gamma$. As a result, the slope of the power-law part of the distribution remains unchanged, but the upper and lower limits shift downwards in energy. Of course, the situation is more complicated if, simultaneously, radiation losses are important. Our treatment of losses in the lobes, where an explicit time dependence of the energy density is available, takes this explicitly into account. Qualitatively, however, the form of the spectrum should not depend on the sequence in which these processes operate. In treating propagation through the head region we impose the adiabatic losses after the radiation losses, bearing in mind that the values derived for quantities such as the diffusion time $t_{\text {diff }}$ may depend somewhat on this choice. Thus, assuming constant injection at the termination shock and requiring particle number conservation, we find for the function describing the injection of electrons into the lobes:

$Q_{\text {lobe }}(\gamma, t)=k Q_{\mathrm{h}}(k \gamma, t)$

where $k(t)=\left[u_{\mathrm{h}} / u_{\text {lobe }}(t)\right]^{1 / 4}$.

Re-acceleration by multiple encounters with shock fronts has been considered in many papers, notably Spruit (1988); Achterberg (1990); Anastasiadis \& Vlahos (1993); Schneider (1993); Pope \& Melrose (1994); Marcowith \& Kirk (1999); Gieseler \& Jones (2000). In our model, we distinguish between the termination shock that forms the primary hot spot and a sequence of subsequent, weaker shocks through which the plasma passes before emerging into the relatively inactive lobe region. The theory of diffusive shock acceleration predicts that a single encounter with a weak shock will not change the power-law index of an incoming distribution produced at a strong shock - the effect is merely to increase the amplitude of the distribution, and to produce a steep power-law tail above the upper cut-off energy of the incoming particles. Passage through many such shocks, however, tends also to harden a preexisting power-law if the ratio of the escape time to the time between shock encounters is large. Shock drift acceleration, on the other hand, produces an effect which is similar to an adiabatic compression (Begelman \& Kirk 1990; Anastasiadis \& Vlahos 1993), in that the power-law index is unchanged, but the distribution is shifted and slightly modified around the cut-off energies. The effect of a highly turbulent environment comprising a mixture of different types of accelerators has been studied by Manolakou et al. (1999) who conclude that the level of turbulent activity has at most a minor impact on a pre-existing power-law index, and contributes only to the efficiency of the process. Given the variety of possible effects, it is clearly a difficult observational challenge to distinguish between the different mechanisms. In this paper, we do not adopt a specific acceleration model, but simply assume that when re-acceleration is important a distribution emerges into the lobe which can be described as a power-law above a lower cut-off energy, modified by synchrotron and inverse Compton losses at high energy. This corresponds precisely to a modification of the effectiveness of the adiabatic losses described by the parameter $k(t)$. If we now interpret the quantity $\eta$ as that fraction of the jet power which goes into energetic particles which enter the lobe, then the effects of re-acceleration correspond simply to setting $k(t) \equiv 1$ in Eq. (20).

\subsection{The evolution of the electron distribution in the lobes}

The spatially integrated distribution $N(\gamma, t)$ of particles in the lobes, is governed by a kinetic equation similar to Eq. (16):

$\frac{\partial N}{\partial t}+\frac{\partial}{\partial \gamma}(\dot{\gamma} N)=Q_{\text {lobe }}(\gamma, t)$.

However, in this case the loss term is more complicated, involving adiabatic losses as well as synchrotron and Compton losses, the first two of which have an explicit time-dependence:

$\dot{\gamma}=-b \frac{\gamma}{t}-b_{\mathrm{ic}} \gamma^{2}-b_{\mathrm{s}} \frac{\gamma^{2}}{t^{a}}$

The three terms on the right hand-side of Eq. (22) represent (i) adiabatic losses, with $b=a / 4$ according to Eq. (5), (ii) losses by inverse Compton scattering, with $b_{\text {ic }}=(4 / 3)\left(\sigma_{\mathrm{T}} / m_{\mathrm{e}} c\right) u_{\mathrm{c}}$ a constant, and (iii) synchrotron losses in the adiabatically decreasing magnetic field of the lobe (see Eq. (5)): $b_{\mathrm{s}}=(4 / 3)\left(\sigma_{\mathrm{T}} / m_{\mathrm{e}} c\right) u_{\mathrm{h}} t_{0}^{a}$.

Following the method of characteristics, Eq. (21) may be rewritten in terms of the derivative of $N$ along a family of curves $\chi(\gamma, t)$ describing the evolution of individual particles and given by integrating Eq. (22):

$\chi(\gamma, t)=\frac{1}{t^{b}}\left(\frac{1}{\gamma}-\frac{b_{\text {ic }} t}{1-b}-\frac{b_{\mathrm{s}} t^{1-a}}{1-b-a}\right)$.

Then $N(\chi, t)$ satisfies the ordinary differential equation

$\frac{\mathrm{d} N}{\mathrm{~d} t}-\left[\frac{b}{t}+2 \gamma\left(b_{\mathrm{ic}}+b_{\mathrm{s}} t^{-a}\right)\right] N=Q_{\text {lobe }}(\gamma, t)$,

in which $\gamma$ is considered a function of $\chi$ and $t$ according to Eq. (23).

Equation (24), with $Q_{\text {lobe }}$ given by Eq. (20), may be integrated numerically, the upper and lower limits of integration being the observation time, $t$ and the earliest time $t_{\mathrm{i}}(\gamma, t)$ at which the source injected particles into the lobes that subsequently cooled to the chosen Lorentz factor $\gamma$ at time $t$. The initial condition is $N\left(\chi, t_{\mathrm{i}}\right)=0$.

The injection time $t_{\mathrm{i}}$ is a function of the Lorentz factor, $\gamma$ and the observing time, $t$. It is determined not only by the cooling rate, but also, at least potentially, by the time $t_{\text {start }}$ at which the source started its activity. This limit is important if particles injected into the lobe at time $t_{\text {start }}$ with $\gamma_{\mathrm{i}}<$ $\gamma_{\max } / k\left(t_{\text {start }}\right)$ can cool to $\gamma$ at time $t$. From Eq. (23), this situation arises when

$\frac{1}{\gamma_{\mathrm{i}}}=t_{\mathrm{start}}\left(\frac{b_{\mathrm{ic}}}{1-b}+\frac{b_{\mathrm{s}} t_{\mathrm{start}}-a}{1-b-a}\right)+t_{\text {start }}^{b} \chi(\gamma, t)<\frac{k\left(t_{\mathrm{start}}\right)}{\gamma_{\max }}$

in which case we set $t_{\mathrm{i}}=t_{\text {start }}$. If, on the other hand, $\gamma_{\mathrm{i}}>$ $\gamma_{\max } / k\left(t_{\text {start }}\right)$, then $t_{\mathrm{i}}$, is found from the relation:

$\frac{k(t)}{\gamma_{\max }}=t_{\mathrm{i}}\left(\frac{b_{\mathrm{ic}}}{1-b}+\frac{b_{\mathrm{s}} t_{\mathrm{i}}^{-a}}{1-b-a}\right)+t_{\mathrm{i}}^{b} \chi(\gamma, t)$.

In summary, the injection time, $t_{\mathrm{i}}$ lies in the range $t_{\mathrm{start}}<t_{\mathrm{i}}<t$, if $\gamma_{\mathrm{i}}>\gamma_{\max } / k\left(t_{\text {start }}\right)$ and equals $t_{\text {start }}$ if $\gamma_{\mathrm{i}}<\gamma_{\max } / k\left(t_{\mathrm{start}}\right)$.

Finally, the specific power $P_{v}(t)$ (power per unit frequency) is found by integrating the product of $N(\gamma, t)$ and the emissivity 

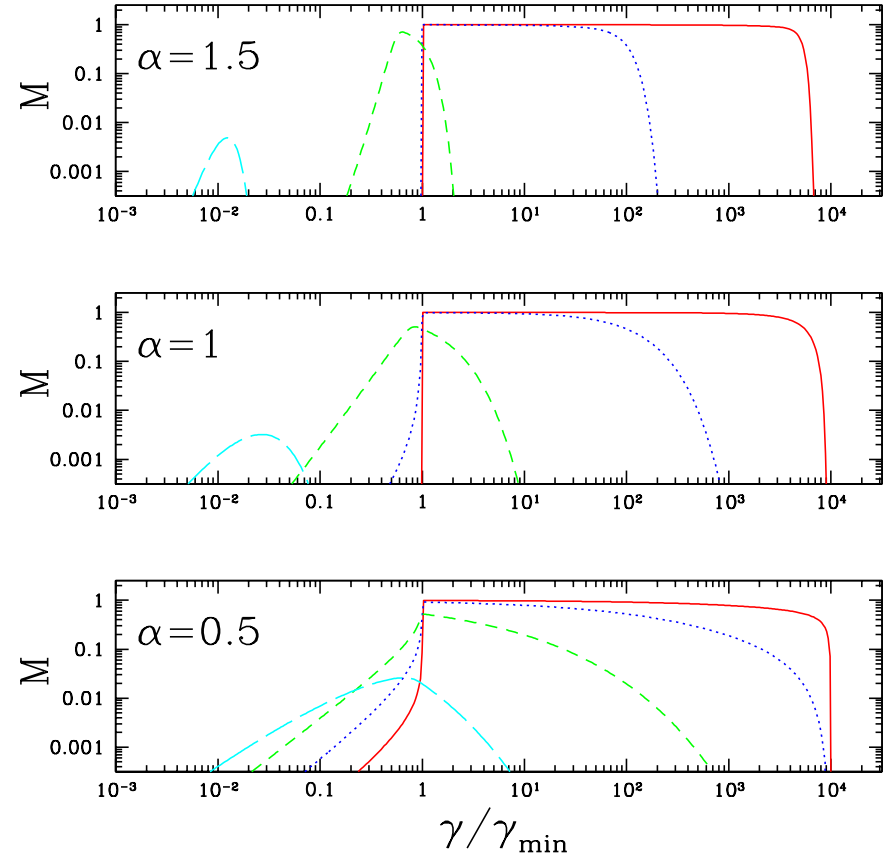

Fig. 2. The modulation function $M\left(\hat{\gamma}, \rho_{\mathrm{d}}, p, \tau\right)$ defined in Eq. (18) as a function of $\hat{\gamma}$, for $\rho_{\mathrm{d}}=10^{-4}, p=2.23$ and for $\alpha=1$ (diffusion), $\alpha=0.5$ (sub-diffusion) and $\alpha=1.5$ (supra-diffusion). In each case the solid, dotted, dashed and long-dashed curves correspond to $\tau=10^{-4}$, $10^{-2}, 1$ and $10^{2}$, respectively.

of a single electron. Adopting the delta-function approximation we have:

$P_{v}(t)=\frac{1}{4 \pi} \int_{1}^{\infty} a_{0} \gamma^{2} B_{\mathrm{L}}(t)^{2} \delta\left(v-a_{1} B_{\mathrm{L}}(t) \gamma^{2}\right) N(\gamma, t) \mathrm{d} \gamma$

where $B_{\mathrm{L}}(t)$ is the magnetic field in the lobe at time $t, a_{0}=$ $1.6 \times 10^{-14} \mathrm{~W} \mathrm{~T}^{-2}$ and $a_{1}=1.3 \times 10^{10} \mathrm{~Hz} \mathrm{~T}^{-1}$.

\section{Tests and special cases}

\subsection{Cooling in the head region}

The effect of cooling in the relatively strong magnetic field of the head region is described by the modulation function $M$ of Eq. (18). This function is plotted in Fig. 2 for various values of the ratio of diffusion to cooling time $\tau$.

The character of the three different transport regimes is clearly seen from this figure. In the case of supra-diffusion, $\alpha>1$, the effects of cooling on the distribution of particles injected at the termination shock are very similar to those calculated by Kardashev (1962) under the assumption that each particle spends the same length of time within the cooling zone. The upper cut-off moves towards lower Lorentz factors as $\tau$ rises, but remains quite sharp. A low energy tail at Lorentz factors less than those injected is almost absent for $\tau<1$, where the cooling time of the lowest energy injected particle is longer than the transport time $t_{\text {diff }}$. For larger values of $\tau$, the particles escaping from the head region have a sharply peaked distribution centred at $\hat{\gamma} \approx 1 / \tau$.
For diffusive transport ( $\alpha=1)$ the spread in escape times is appreciable, resulting in a smoother cut-off and earlier development of a low energy tail at Lorentz factors $\hat{\gamma}<1$. Results for this case have been presented by Wilson (1975) and Valtaoja (1984). In sub-diffusion $(\alpha<1)$ these trends are even stronger. Even for the relatively fast transport through the cooling region implied by $\tau=10^{-2}$, the particle spectrum is steepened significantly over the entire injected range from $\hat{\gamma}=1$ to $\hat{\gamma}=10^{4}$. At low Lorentz factors a power-law tail is noticeable already at $\tau=10^{-4}$. The very large spread in escape times implied by this model can be seen from the distribution plotted for $\tau=100$. A substantial number of particles leave the head region with Lorentz factors of $\hat{\gamma}=10$. These particles have escaped within $10^{-3}$ of the characteristic transport time $t_{\text {diff }}$. On the other hand, the distribution for $\tau=10^{-4}$ reveals particles escaping with $\hat{\gamma}=0.2$, which have been retained in the head region for over $10^{4} \times t_{\text {diff }}$.

In summary, the main effect of cooling in the high-loss head region on the particle distribution is not to steepen it into a new power-law, but to impose a cut-off of varying degrees of sharpness, according to the transport properties. The adiabatic expansion losses suffered by these particles before entering the lobes shifts the escaping spectrum in energy and amplitude, but does not change its form.

\subsection{The lobe}

The effect of synchrotron and inverse Compton losses that are constant in time on a power-law distribution $Q_{\text {lobe }} \propto \gamma^{-s}$ of injected electrons is well known. However, losses in combination with time-dependent adiabatic expansion and continuous injection - processes present in Eq. (21) - considerably complicate this simple picture. Analytic solutions can be found for the regimes in which one or other of these processes dominates. Two of these are given in the Appendix and are briefly discussed in this section. In each case it is assumed that a power-law distribution is continuously injected in the range $\gamma_{1}<\gamma<\gamma_{2}$.

\subsubsection{Adiabatic losses}

In this case Eq. (22) becomes $\dot{\gamma}=-b \gamma / t$. The solution of Eq. (21) is given by Eq. (29). It is characterised by a range over which the distribution has the same slope as the injection function $N \propto \gamma^{-s}$ and accumulates approximately linearly with time. Above a critical value of the Lorentz factor, which decreases with time, the distribution cuts off, tending to zero at $\gamma_{2}$. Below $\gamma_{1}$, a power-law distribution is established with $N \propto-1+1 / b$ which also accumulates approximately linearly with time. (In the cases of relevance here, $b \approx 0.4$.) The distribution vanishes below a lower cut-off, that decreases with time.

\subsubsection{Inverse Compton losses}

In this case Eq. (22) becomes $\dot{\gamma}=-b_{\text {ic }} \gamma^{2}$, and the solution of Eq. (21) is given in Eq. (30). Below a time-dependent break frequency the distribution has the same slope as the injection 
function $N \propto \gamma^{-s}$ and accumulates steadily with time. Above the break frequency the distribution steepens by unity: $N \propto$ $\gamma^{-s-1}$ and remains constant in time, finally cutting off at the maximum injected Lorentz factor $\gamma_{2}$. For $\gamma<\gamma_{1}$ a cooling distribution $N \propto \gamma^{-2}$ is established.

Examples of these properties can be seen in Fig. 3, which shows the evolution of the normalised electron distribution, $N(\gamma, t)$ for the case of a high jet-power, high redshift radio source. The continuous lines represent the numerical results and the crosses (where available) the analytical solutions, as described in Eqs. (29) and (30). The values of the parameters used in this case are: jet power $Q_{0}=1.3 \times 10^{40} \mathrm{~W}$, source redshift $z=2$, hotspot radius $R=2.5 \mathrm{kpc}, \gamma_{1}=10$ and $\gamma_{2}=10^{7}$. The timescale associated with these parameters is $t_{0}=2.5 \times 10^{5} \mathrm{yr}$ and the source activity is assumed to start at $t_{\text {start }}=t_{0}$; our numerical results are insensitive to this choice. The efficiency was chosen to be $\eta=10^{-1}$.

The amplitude of the energy spectrum increases with time since the rate at which particles enter the lobe is higher than the rate at which they cool to lower energies. At epochs earlier than $\left(b_{\mathrm{s}} / b_{\text {ic }}\right)^{1 / a}=t_{\text {crit }} \approx 21 \mathrm{Myr}$ synchrotron losses dominate over Compton losses at all energies. Adiabatic losses dominate below a critical Lorentz factor given by $\gamma_{\text {crit }}=b t^{a-1} / b_{\mathrm{s}} \approx$ $10^{2}[t(\mathrm{Myr})]^{0.57}$ at early times $\left(t<t_{\text {crit }}\right)$ and by $\gamma_{\text {crit }}=b / b_{\text {ic }} t \approx$ $10^{4} / t$ (Myr) at late times $\left(t>t_{\text {crit }}\right)$. Thus, in Fig. 3 , the distribution at $1 \mathrm{Myr}$ is dominated by adiabatic losses for $\gamma<10^{2}$ and by (time dependent) synchrotron losses for $\gamma>10^{2}$. At epochs $100 \mathrm{Myr}$ and $200 \mathrm{Myr}$ Compton losses dominate above $\gamma=100$ and 50 respectively. Because the synchrotron losses decrease with time, even the loss-dominated part of the distribution grows significantly between 1 and $10 \mathrm{Myr}$. When Compton losses are decisive, the loss dominated part remains almost constant. Below $\gamma=10$ (the minimum Lorentz factor at which injection occurs) a power-law distribution of index $-1+1 / b \approx 1.6$ is established by adiabatic losses.

\section{Results}

In this section we present some results concerning the spectral evolution of the lobes of classical double radio sources, derived by integrating the electron energy distribution in the lobes, $N(\gamma, t)$, over the electron emissivity given in Eq. (27). The electron energy distribution is computed numerically by integrating Eq. (24) using the differential rate at which electrons are injected into the lobe given in Eqs. (20) and (18).

The parameters we use to specify a radio source are:

1. The jet power $Q_{0}$.

2. The efficiency $\eta(\leq 1)$ with which particles are accelerated at the terminal shock front, which we set to unity in case A (without re-acceleration), and to 0.4 in case B (with re-acceleration). The specific power is linearly proportional to $\eta$.

3. The area $A$ of the terminal hot spot - we take $A=\pi \times$ $(2.5)^{2} \mathrm{kpc}^{2}$.

4. The redshift $z$ of the source

5. The density of the surrounding matter at a fiducial distance, according to Eq. (1).
6. The density profile, for which we take $\beta=1.5$.

7. The power-law index $p$ of particles accelerated at the terminal shock. Depending on the Lorentz factor of the jet, one expects $p \approx 2$ to 2.3. We adopt the value 2.23 (Kirk et al. 2000).

8. The minimum Lorentz factor of the accelerated particles. This should be somewhat larger than the Lorentz factor of the jet. The results are not sensitive to its value for the relevant range of spectral indices.

9. The ratio $\tau$ of the typical transport time $t_{\text {diff }}$ to cooling time $t_{\text {cool }}$ at $\gamma=\gamma_{\min }$. This is essentially a free parameter. Although spherical geometry is used to motivate the transport model in Sect. 3, the resulting concept of a stochastic spread in particle escape times from the high magnetic field head region is much more generic. However, as in the case of standard diffusion, the model assumes infinite velocity of a fluid element within the head region. This could lead to unphysical results if $t_{\text {diff }}$ is chosen to be too small. In our case, if $R_{\mathrm{esc}}$ is the minimum distance a fluid element must travel before exiting the head region then the ratio of escape time to $t_{\text {cool }}$ must exceed $10^{-4} \gamma_{\text {min }}\left(Q / 10^{40} \mathrm{~W}\right)\left(R_{\text {esc }} / 1 \mathrm{kpc}\right)$ for all particles. In the case of sub-diffusion, where the individual escape times have a large spread about the mean, $\tau$ must exceed this value by a factor of a few. Thus, in our computations we take $\tau \geq 2 \times 10^{-3}$.

10. The maximum Lorentz factor $\gamma_{\max }$ to which particles are accelerated at the terminal shock. In accordance with X-ray observations of hot spots we assume $\gamma_{\max } \approx 10^{7}$. As a result, $\tau \gg \gamma_{\min } / \gamma_{\max }$ and the results are insensitive to this parameter.

11. The index $\alpha$ that characterises the transport process through the head region: $0<\alpha<2$. We consider three values: sub-diffusion $\alpha=0.5$, diffusion $\alpha=1$ and supra diffusion $\alpha=1.5$.

In addition we distinguish two cases:

- Case A: particles suffer time-dependent adiabatic and radiative losses on passing from the termination shock to the lobes.

- Case B: adiabatic losses between the termination shock and the lobes are compensated by re-acceleration in the head region. In this case $k(t) \equiv 1$ in Eq. (20).

We consider first tracks on the power versus linear size diagram of high power, high redshift sources. Figure 4 shows two such tracks for case A - no re-acceleration of particles after the termination shock - for parameters given in Table 1 . Three different transport models are shown $(\alpha=0.5,1$, and 1.5 ) and we have chosen $\tau=2 \times 10^{-3}$. This figure also shows the positions of FR II sources in the $3 \mathrm{CR}$ revised catalogue, (Laing et al. 1983) according to the classifications by Jackson \& Rawlings (1997), as updated and used by Willott et al. (1999) and published on-line by Willott (2002). The linear size was computed assuming the cosmological parameters $q_{0}=0$, $H_{0}=50 \mathrm{~km} \mathrm{~s}^{-1} \mathrm{Mpc}^{-1}$. Sources with redshift $z>0.5$ are indicated by a cross, those with $z<0.5$ by an open circle.

The first track, labelled "standard" can be directly compared with Fig. 13 of Blundell et al. (1999), to reveal the new 

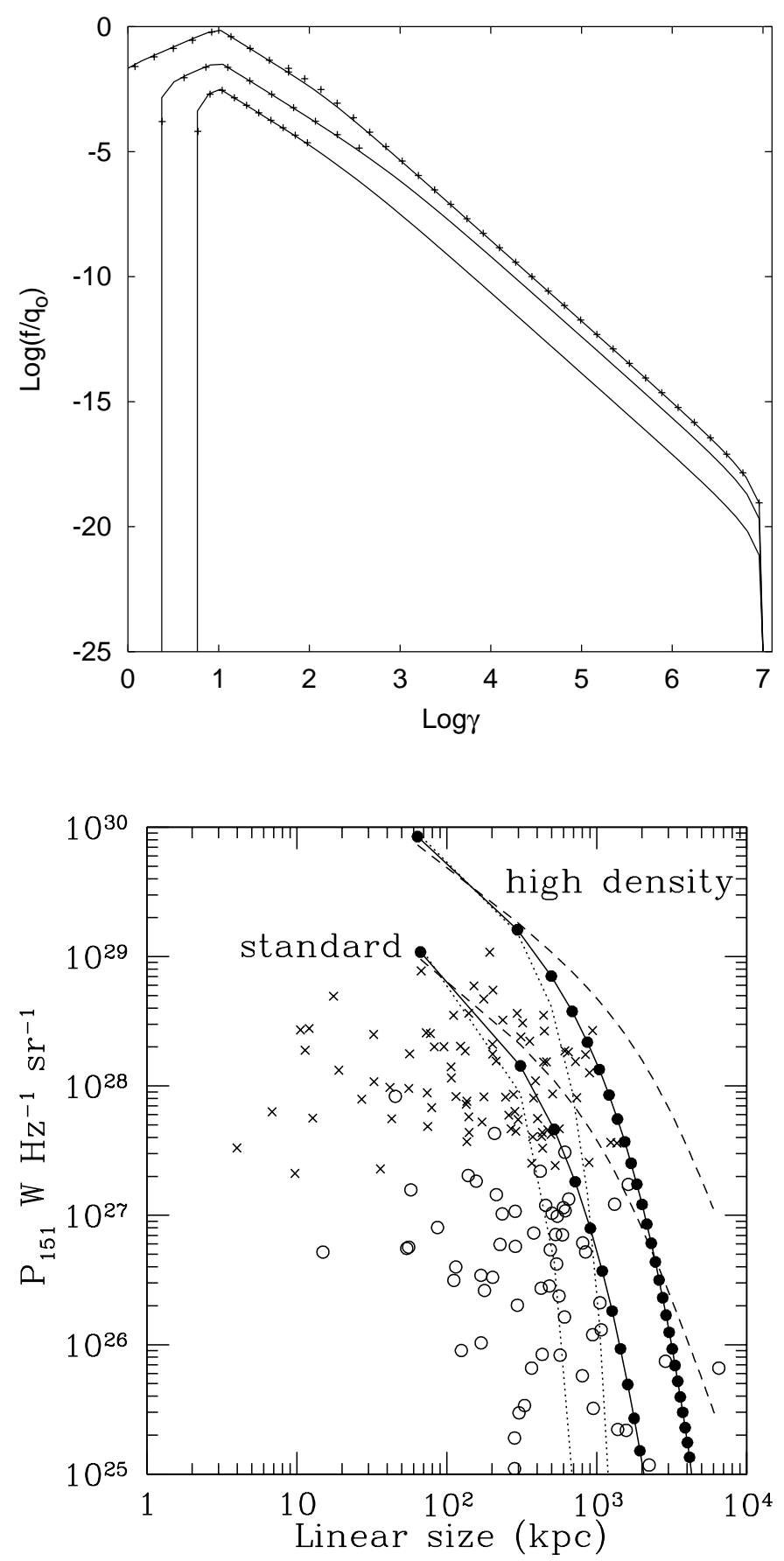

features of our model. We find a much sharper decline in the source power with increasing radius than predicted by Blundell et al. (1999). For these computations we adopt the minimum plausible value of the transport time: $\tau=2 \times 10^{-3}-$ increasing $\tau$ always leads to an even steeper decline. The track passes well below the large $(D>600 \mathrm{kpc})$ sources with specific power $P_{151}>3 \times 10^{28} \mathrm{~W} \mathrm{~Hz}^{-1}$ and decays to $P_{151} \approx 7 \times 10^{26} \mathrm{~W} \mathrm{~Hz}^{-1}$ within less than $26 \mathrm{Myr}$. The reason for this behaviour is the lack of particles of high Lorentz factor entering the lobes. Synchrotron losses in the head region effect a cut-off of the injected distribution in our model of acceleration and transport. After suffering the strong expansion losses associated with the
Fig. 3. The evolution of the electron distribution in the lobe of a high jet power high redshift radio source, in the case of a power-law injection spectrum from the hotspot. From bottom to top: $t=1,10$ and $200 \mathrm{Myr}$, respectively. Continuous lines represent the numerical results and crosses the analytical solutions as described by Eqs. (29) and (30).

Fig. 4. Tracks for two high redshift, high power sources for case A (no re-acceleration), with parameters as given in Table 1. The "standard" track corresponds to Blundell et al. (1999), Fig. 13. On each track the dots indicate the source age, starting at $1 \mathrm{Myr}$ and uniformly spaced in intervals of $5 \mathrm{Myr}$. The solid line shows the track for diffusion $(\alpha=1)$ the dashed line for sub-diffusion $(\alpha=0.5)$ and the dotted line for supra-diffusion $(\alpha=1.5)$. Crosses and open circles indicate FR II sources of the 3CR revised sample, with $z>0.5$ and $z<0.5$, respectively.

pressure difference between primary hot spot and lobe, this cut-off affects the number of particles available to radiate at $151 \mathrm{MHz}$, which, as a result, declines steeply with age. In order to account for the larger sources, it is necessary not only to assume a much larger power $Q_{0} \approx 6.5 \times 10^{40} \mathrm{~W}$, but also a much higher surrounding density $\rho \approx 10^{-22} \mathrm{~kg} \mathrm{~m}^{-3}$ at $10 \mathrm{kpc}$. Such a track is shown in Fig. 4 labelled "high density". At age roughly $22 \mathrm{Myr}$ this track coincides with the largest high power source in the 3CR revised catalogue. If sources existed with these extreme parameters they should also be visible at much higher specific powers, when less than 10 Myr old. Such objects, however, do not appear in the catalogue. We therefore conclude that 
Table 1. Parameters of the models displayed in Figs. 4 and 5.

\begin{tabular}{lllll}
\hline \hline & $Q_{0}(\mathrm{~W})$ & $\eta$ & $z$ & $\begin{array}{c}\rho\left(\mathrm{kg} \mathrm{m}^{-3}\right) \\
\text { at } 10 \mathrm{kpc}\end{array}$ \\
\hline Fig. 4, "standard" & $1.3 \times 10^{40}$ & 1. & 2. & $1.7 \times 10^{-23}$ \\
Fig. 4, "high density" & $6.5 \times 10^{40}$ & 1. & 2. & $1 . \times 10^{-22}$ \\
Fig. 5, "high $z$ " & $1.3 \times 10^{40}$ & 0.4 & 2. & $1.7 \times 10^{-23}$ \\
Fig. 5, "low $z "$ & $1.3 \times 10^{38}$ & 0.4 & 0.2 & $1.7 \times 10^{-23}$ \\
\hline
\end{tabular}

case A tracks, in which particle acceleration occurs only at the termination shock are in conflict with the data.

This conclusion is unaffected by the type of transport present in the head region. The dashed lines in Fig. 4 show the effects of sub-diffusion and the dotted lines that of supra-diffusion on the "standard" and "high density" tracks. For ages greater than $5 \mathrm{Myr}$, sub-diffusion always results in greater specific power than diffusion $(\alpha=1)$, whereas supra-diffusion gives a lower specific power. This arises because sub-diffusion produces a greater spread in escape times from the head region than does diffusion. The upper cut-off in the Lorentz factor of particles entering the lobes is correspondingly higher, whereas the opposite is the case for supradiffusion. Nevertheless, the sub-diffusion track for the standard source is unable to account for the largest high redshift sources, and the supra-diffusion track for a high density source would predict more medium-sized high specific power sources than are observed.

Turning to case $\mathrm{B}$, in which the adiabatic losses between head region and lobe are compensated by a re-acceleration process operating downstream of the termination shock, we present in Fig. 5 two tracks in the $P-D$ diagram, one corresponding to a high redshift, high power source and one to a low redshift, low power source. Once again, the parameters are chosen to enable direct comparison with the work of (Blundell et al. 1999) and are shown in Table 1. The value of $\tau$ is the same as in Fig. 4.

A prominent feature of these tracks is the steepening as the source ages. To a lesser extent, this is also seen in case A, Fig. 4, and is present in the tracks computed by Kaiser et al. (1997) and Blundell et al. (1999). It arises at the point where inverse Compton losses become important in the lobes. Whereas the synchrotron losses continually weaken with time, the inverse Compton losses remain constant, causing a more dramatic fall off in the specific power.

Because of the dependence of the source size on its power (see Eq. (3)) the low $z$ source in Fig. 5 is systematically older at a fixed source size than is the high $z$ source - a potentially important fact in the interpretation of the correlation between source size and spectral index. In Fig. 6 we plot the particle distribution in the lobes, multiplied by $\gamma^{p}$, against Lorentz factor $\gamma$ for both the high $z$ source (solid line) and the low $z$ source (dashed line). Despite the age difference, it can be seen that where cooling steepens the spectrum, the high power, high $z$ source always has a spectrum that is softer than that of the low power, low $z$ source. Furthermore, for fixed redshift, the source spectrum systematically softens with increasing size, and, therefore, also with increasing age.

\section{Conclusions}

We have introduced a new description of the acceleration and transport of particles in the hot spots and lobes of FR II radio galaxies. Using the standard approach of assuming a power-law spectrum injected at the termination shock, we model the subsequent transport through the head region into the lobes using a formalism which permits the investigation of "anomalous" regimes. These transport regimes are described by the single parameter $\alpha$, which determines the time-dependence of the mean-square displacement of a particle: $\left\langle\Delta r^{2}\right\rangle \propto t^{\alpha}$. Standard diffusion, in which the flux is proportional to the gradient of the particle density (Fick's law) corresponds to $\alpha=1$. The anomalous regimes of sub-diffusion and supra-diffusion correspond respectively to $\alpha<1$ and $\alpha>1$. In the current application, the most important physical difference between these regimes is that they permit a range of escape times for the particles from the high-loss head region. Compared to the case of standard diffusion, these times have a much wider distribution in subdiffusion and a much tighter one in supra-diffusion. The new formalism also requires a parameter $\tau$ that corresponds to the average residence time in units of the cooling time. Thus, in total, one more parameter is used than the standard Kardashev (1962) model with a single escape time for all particles and one parameter fewer is needed than the model introduced by Blundell et al. (1999), which requires two break frequencies and a "leakiness".

The self-similar description of the hydrodynamical evolution of classical double radio sources implies that the lobe pressure falls with time. Because the pressure in the primary hot-spots does not appear to undergo this evolution, the particles injected at there in older sources have to overcome ever more severe adiabatic losses. These losses were not modelled by Kaiser et al. (1997), who specified the distribution of particles entering the lobes. Blundell et al. (1999) pointed out their importance and included them in their $P-D$ tracks. However, because the transport model did not include an upper cut-off of injected particles, but merely a spectral break, they were still able to account for the observed population of sources. We find that the adiabatic losses between primary hot spot and lobe prevent this when our transport model is adopted. The large, high luminosity sources can be explained only as very powerful jets in very dense environments, in which case they should have smaller, brighter predecessors, that are absent from the $3 \mathrm{CR}$ revised catalogue. There could be several reasons for this. One possibility is that at high redshift, small sources with powerful jets exist, but are not bright in the radio because of absorption. The missing progenitors could then be powerful, high redshift analogues of the "Gigahertz Peak Spectrum" sources (Bicknell et al. 1997). Another is that the jet power $Q_{0}$ remains quite weak during the early evolution of a high-redshift source, becoming strong only after $\sim 10 \mathrm{Myr}$. Intermittent jet activity in FR II sources has already been proposed (Reynolds \& Begelman 1997), but as a solution to the opposite problem of an observed over-abundance of small powerful sources 

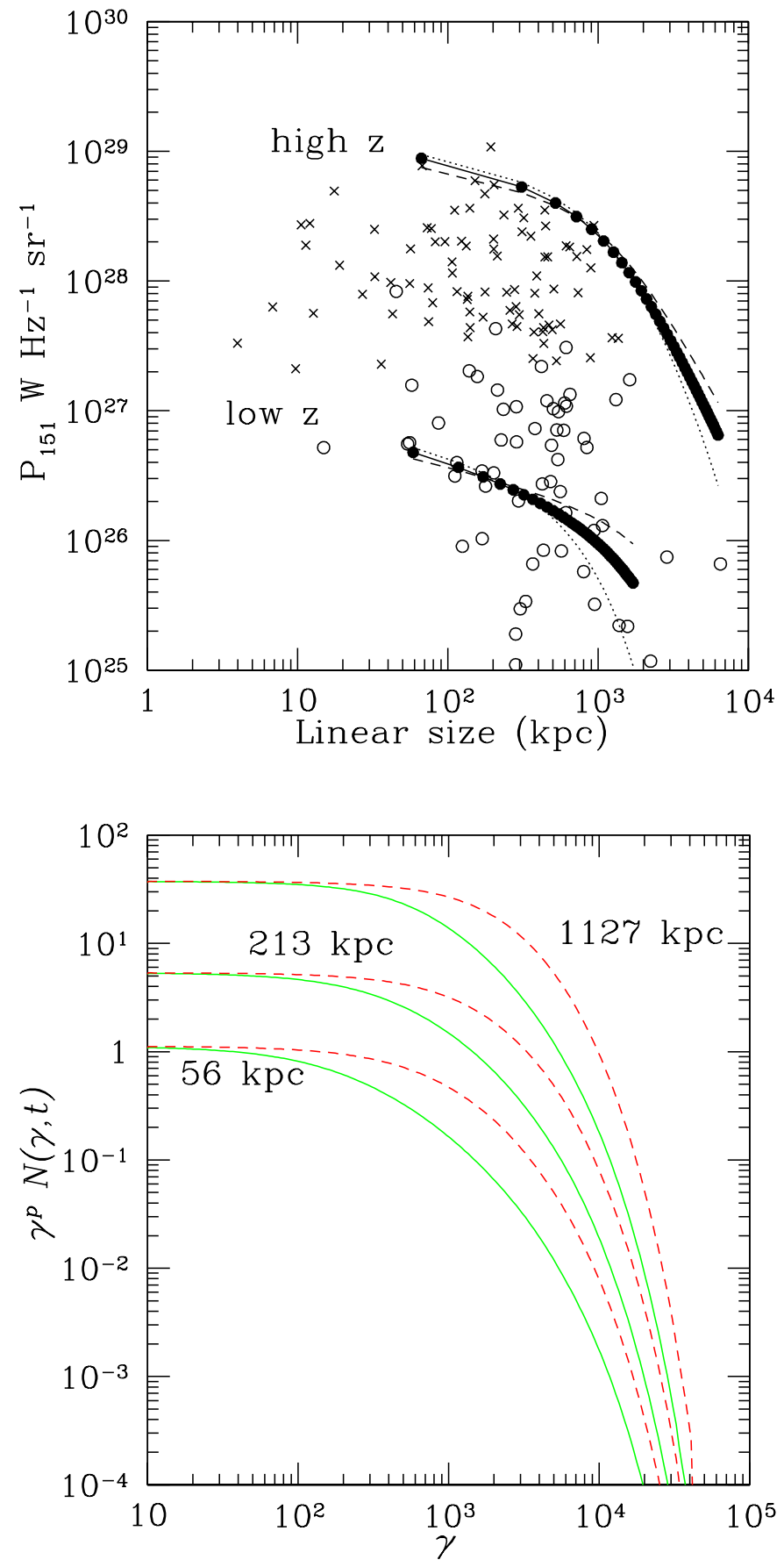

(at lower redshift). In general, it seems easier to imagine the jet power to be larger during earlier phases of a source's life, leading to a faster decay of the specific source power with time.

However, the solution we propose to this problem is that acceleration is not confined to the primary hot spot, but occurs throughout the head region. Independent evidence from individual sources in the infra-red, optical and X-ray bands (Meisenheimer et al. 1996, 1997; Perley et al. 1997; Wagner \& Krawczynski 2000) supports this idea. A plausible mechanism is repeated encounters with weaker shock fronts which may permeate this turbulent region. We have investigated the $P-D$ tracks and spectra of a model in which it is assumed that this distributed acceleration compensates the expansion losses
Fig. 5. Tracks for a high redshift, high power source and a low redshift, low power source for case B (including re-acceleration), with parameters as given in Table 1. Diffusive transport $\alpha=1$ is shown by a solid line, on which the source age is indicated by the dots, starting, for the high $z$ source, at $1 \mathrm{Myr}$ and, for the low- $z$ source, at 4 Myr and uniformly spaced in intervals of $5 \mathrm{Myr}$ in each case. The dashed lines indicate sub-diffusion $\alpha=0.5$ and the dotted lines supra-diffusion $(\alpha=1.5)$ Crosses and open circles indicate FR II sources of the 3CR revised sample, with $z>0.5$ and $z<0.5$, respectively.

Fig. 6. The particle distribution function in the lobes, multiplied by $\gamma^{p}$, where $p$ is the index of the injected distribution for the high and low redshift sources shown in Fig. 5. The solid lines depict the distribution for the high $z$ source, the dashed lines for the low $z$ source. For each source the distribution is plotted when it achieves the sizes 56, 213 and $1127 \mathrm{kpc}$. For fixed size, the higher redshift source has the softer spectrum. For fixed redshift, the older (larger) source is softer.

between primary hot spot and lobes, without significantly modifying the spectrum. Our tracks are more akin to those presented by Kaiser et al. (1997) and we have investigated their sensitivity to the new transport parameter $\alpha$. We have shown that some of the properties noted by Blundell et al. (1999), such as a redshift/spectral index and a size/spectra index correlation also emerge from our model.

Acknowledgements. We thank Klaus Meisenheimer and Stefan Wagner for helpful discussions and Jane Dennett-Thorpe for a careful reading of the manuscript and valuable suggestions. K.M. acknowledges support from the German Research Council (DFG) under SFB 439. 


\section{Appendix}

Analytic solutions of Eq. (21) are presented for the cases in which either only adiabatic losses or only synchrotron losses are present, assuming an injection function given by

$Q_{\text {lobe }}(\gamma)= \begin{cases}q_{0} \gamma^{-s} & \text { for } \gamma_{1} \leq \gamma \leq \gamma_{2} \\ 0 & \text { otherwise }\end{cases}$

\subsection{Adiabatic losses}

The solution of Eq. (21), provided that $\gamma_{1}<\gamma_{2}\left(t / t_{\text {start }}\right)^{-b}$, is:

$f(\gamma, t)= \begin{cases}0, & \gamma<\gamma_{a_{1}} \\ \frac{q_{0} t_{\text {start }} \gamma^{-s}}{\lambda}\left(\frac{\gamma}{\gamma_{1}}\right)^{\frac{\lambda}{b}}\left(\frac{t}{t_{\text {start }}}\right)\left\{1-\left[\left(\frac{\gamma}{\gamma_{1}}\right)\left(\frac{t}{t_{\text {start }}}\right)^{b}\right]^{-\frac{\lambda}{b}}\right\}, & \gamma_{a_{1}} \leq \gamma \leq \gamma_{1} \\ \frac{q_{0} t_{\text {start }} \gamma^{-s}}{\lambda}\left(\frac{t}{t_{\text {start }}}\right)\left[1-\left(\frac{t}{t_{\text {start }}}\right)^{-\lambda}\right], & \gamma_{1}<\gamma<\gamma_{a_{2}} \\ \frac{q_{0} t_{\text {start }} \gamma^{-s}}{\lambda}\left(\frac{t}{t_{\text {start }}}\right)\left[1-\left(\frac{\gamma}{\gamma_{2}}\right)^{\frac{\lambda}{b}}\right], & \gamma_{a_{2}} \leq \gamma \leq \gamma_{2} \\ 0, & \gamma>\gamma_{2}\end{cases}$

with $\lambda=b(s-1)+1$ and $\gamma_{a_{1}}=\gamma_{1}\left(t / t_{\text {start }}\right)^{-b}, \gamma_{a_{2}}=\gamma_{2}\left(t / t_{\text {start }}\right)^{-b}$.

The solution of Eq. (21), for $\gamma_{1}<\left[\gamma_{2}^{-1}+b_{\text {ic }}\left(t-t_{\text {start }}\right)\right]^{-1}$, is:

$f(\gamma, t)= \begin{cases}0, & \gamma<\gamma_{c_{1}} \\ K \gamma^{-(s+1)}\left\{\left(\frac{\gamma}{\gamma_{1}}\right)^{s-1}\left[1-b_{\text {ic }} \gamma\left(t-t_{\text {start }}\right)\right]^{s-1}\right\}, & \gamma_{c_{1}} \leq \gamma \leq \gamma_{1} \\ K \gamma^{-(s+1)}\left\{1-\left[1-b_{i c} \gamma\left(t-t_{\text {start }}\right)\right]^{s-1}\right\}, & \gamma_{1}<\gamma<\gamma_{c_{2}} \\ K \gamma^{-(s+1)}\left[1-\left(\frac{\gamma}{\gamma_{2}}\right)^{s-1}\right], & \gamma_{c_{2}} \leq \gamma \leq \gamma_{2} \\ 0, & \gamma>\gamma_{2}\end{cases}$

where $K=q_{0} / b_{\text {ic }}(s-1), \gamma_{\mathrm{c}_{1}}=\left[\gamma_{1}^{-1}+b_{\text {ic }}\left(t-t_{\text {start }}\right)\right]^{-1}$ and $\gamma_{\mathrm{c}_{2}}=\left[\gamma_{2}^{-1}+b_{\text {ic }}\left(t-t_{\text {start }}\right)\right]^{-1}$.

In the case that adiabatic losses determine the spectrum of a source the solution of Eq. (21) for $\gamma_{1}>\gamma_{a_{2}}$, is:

$f(\gamma, t)= \begin{cases}0, & \gamma<\gamma_{a_{1}} \\ \frac{q_{0} t_{\text {start }} \gamma^{-p}}{\lambda}\left(\frac{\gamma}{\gamma_{1}}\right)^{\frac{\lambda}{b}}\left(\frac{t}{t_{\text {start }}}\right)\left\{1-\left[\left(\frac{\gamma}{\gamma_{1}}\right)\left(\frac{t}{t_{\text {start }}}\right)^{b}\right]^{-\frac{\lambda}{b}}\right\}, & \gamma_{a_{1}} \leq \gamma \leq \gamma_{a_{2}} \\ \frac{q_{0} t_{\text {start }} \gamma^{(1-b) / b}}{\lambda}\left(\frac{t}{t_{\text {start }}}\right) \gamma_{1}-\lambda / b\left[1-\left(\frac{\gamma_{1}}{\gamma_{2}}\right)^{\lambda / b}\right], & \gamma_{a_{2}}<\gamma \leq \gamma_{1} \\ \frac{q_{0} t_{\text {start }} \gamma^{-p}}{\lambda}\left(\frac{t}{t_{\text {start }}}\right)\left[1-\left(\frac{\gamma}{\gamma_{2}}\right)^{\frac{\lambda}{b}}\right], & \gamma_{1} \leq \gamma \leq \gamma_{2} \\ 0, & \gamma>\gamma_{2} .\end{cases}$

In the case that inverse compton losses determine the spectrum of a source the solution of Eq. (21) for $\gamma>\gamma_{c_{2}}$, is:

$$
f(\gamma, t)= \begin{cases}0, & \gamma<\gamma_{c_{1}} \\ K \gamma^{-2}\left\{\gamma_{1}^{1-p}-\left[\gamma^{-1}-b_{\text {ic }}\left(t-t_{\text {start }}\right)\right]^{p-1}\right\}, & \gamma_{c_{1}} \leq \gamma \leq \gamma_{c_{2}} \\ K \gamma^{-2}\left(\gamma_{1}^{1-p}-\gamma_{2}^{1-p}\right), & \gamma_{c_{2}}<\gamma<\gamma_{1} \\ K \gamma^{-(p+1)}\left[1-\left(\frac{\gamma}{\gamma_{2}}\right)^{p-1}\right], & \gamma_{1} \leq \gamma \leq \gamma_{2} \\ 0, & \gamma>\gamma_{2} .\end{cases}
$$




\section{References}

Achterberg, A. 1990, A\&A, 231, 251

Achterberg, A., Gallant, Y. A., Kirk, J.G., \& Guthmann, A. W. 2001, MNRAS, 328, 393

Alexander, P. 1987, MNRAS, 255, 27

Alexander, P., \& Leahy, J. P. 1987, MNRAS, 225, 1,

Anastasiadis, A., \& Vlahos, L. 1993, A\&A, 275, 427

Blundell, K. M., Rawlings, S., \& Willott, C. J. 1999, AJ, 117, 677

Begelman, M. C., \& Cioffi, D. F. 1989, ApJ, 345, L21

Begelman, M. C., \& Kirk, J. G. 1990, ApJ, 353, 66

Bicknell, G. V., Dopita, M. A., \& O’Dea, C. P. O. 1997, ApJ, 485, 112

Bouchaud, J. P., \& Georges, A. 1990, Phys. Rep., 195, 127

Burch, S. F. 1977, MNRAS, 180, 623

Carilli, C. L., Perley, R. A., Dreher, J. W., \& Leahy, J. P. 1991, ApJ, 383,554

Cox, C. I., Gull, S. F., \& Scheuer, P. A. G. 1991, MNRAS, 252, 558

Falle, S. A. E. G. 1991, MNRAS, 250, 581

Fanaroff, B. L., \& Riley, J. M. 1974, MNRAS, 167, 31

Garrington, S. T., \& Conway, R. G. 1991, MNRAS, 250, 198

Gieseler, U. D. J., \& Jones, T. W. 2000, A\&A, 357, 1133

Jackson, N., \& Rawlings, S. 1997, MNRAS, 286, 241

Jaffe, W. J., \& Perola, G. C. 1973, A\&A, 26, 423

Jagers, W. J. A\&AS, 71, 75, 1987

Kaiser, C. R., \& Alexander, P. 1997, MNRAS, 286, 215

Kaiser, C. R., Dennett-Thorpe, J., \& Alexander, P. 1997, MNRAS, 292,723

Kardashev, N. S. 1962, Soviet Astr. -AJ, 6, 317

Kirk, J. G., Guthmann, A. W., Gallant, Y. A., \& Achterberg, A. 2000, ApJ, 542, 235

Komissarov, S. S., \& Falle, S. A. E. G. 1998, MNRAS, 297, 1087

Laing, R. A., Riley, J. M., \& Longair, M. S. 1983, MNRAS, 204, 151

Liu, R., Pooley, G., \& Riley, J. M. 1992, MNRAS, 257, 545
Manolakou, K., Anastasiadis, A., \& Vlahos, L. 1999, A\&A, 345, 653

Marcowith, A., \& Kirk, J. G. 1999, A\&A, 347, 391

Meisenheimer, K., Neuman, M., \& Röser, H. J. 1996, in Jets from stars and galactic nuclei, ed. W. Kundt, Springer Lecture Notes (Springer-Verlag, Berlin)

Meisenheimer, K., Yates, M. G., \& Röser, H. J. 1997, A\&A, 325, 57

Mulchaey, J. S., \& Zabludoff, A. I. 1998, ApJ, 496, 73

Myers, S. T., \& Spangler, S. R. 1985, ApJ, 291, 52

Pacholczyk, A. G. 1970, Radio Astrophysics (Freeman, San Francisco)

Peacock, J. A. 1999, Cosmological Physics (Cambridge University Press, Cambridge, UK), 431

Perley, R. A., Röser, H. J., \& Meisenheimer, K. 1997, A\&A, 328, 12

Pope, M. H., Ball, L. T., \& Melrose, D. B. 1996, Publ. Astron. Soc. Austr., 13, 132

Pope, M. H., \& Melrose, D. B. 1994, Publ. Astron. Soc. Austr., 11, 175

Ragot, B. R., \& Kirk, J. G. 1997, A\&A, 327, 432

Reynolds, C. S., \& Begelman, M. C. 1997, ApJ, 487, L135

Scheuer, P. A. G. 1982, in Extragalactic Radio Sources, ed. D. S. Heeschen, \& C. M. Wade (Reidel, Dordrecht), IAU Symp., 97, 163

Scheuer, P. A. G., \& Williams, P. J. S. 1968, ARA\&A, 6, 321

Schneider, P. 1993, A\&A, 278, 315

Spruit, H. C. 1988, A\&A, 194, 319

Stephens, P. W. 1987, Ph.D. Thesis, University of Manchester Valtaoja, E. 1984, A\&A, 135, 141

Wagner, S. J., \& Krawczynski, H. 2000, AAS HEAD Meet., 32, 0804

Willott, C. J. 2002 http://www-astro.physics.ox.ac.uk/ cjw/3crr/3crr.html.

Willott, C. J., Rawlings, S., Blundell, K. M. \& Lacy, M. 1999, MNRAS, 309, 1017

Wilson, A. S. 1975, A\&A, 43, 1 